\title{
ESTUDIO DE LA SOLDABILIDAD Y CORROSIÓN DEL ACERO INOXIDABLE AISI 904L CON LOS AGENTES UTILIZADOS EN LA LIXIVIACIÓN DEL COBRE
}

\author{
Ramón Cortés P. ${ }^{1} \quad$ Jaime Villanueva A. $^{2} \quad$ Ernesto Ponce L. $^{3} \quad$ Manuel Rojas M. $^{2} \quad$ Eduardo Rojas Z. ${ }^{2}$ \\ Recibido el 26 de mayo del 2004, aceptado el 21 de septiembre de 2004
}

\begin{abstract}
RESUMEN
La alta agresividad de las soluciones utilizadas en el proceso de lixiviación del cobre y los cuidados especiales que se debe tener para evitar la formación de fases sensibles a estos agentes en la soldadura de aceros inoxidables, ha exigido el desarrollo de nuevos aceros inoxidables que sean más resistentes a la corrosión, manteniendo las propiedades de resistencia a la tracción, al impacto y ductilidad. Es el caso de acero inoxidable AISI 904L, un acero super austenítico de última generación, del cual no se tienen antecedentes de su soldabilidad. En este estudio se verifica su soldabilidad y se determinan los electrodos comerciales más adecuados que minimizan la formación de la fase sigma, fase que produce estructuras cristalinas altamente sensibles a los agentes corrosivos y que mantienen las propiedades del acero analizado.
\end{abstract}

Palabras claves: Soldadura inoxidable, super austenítico, minería.

\begin{abstract}
The high corrosion aggressiveness of solutions used in the copper lixiviation process and the special care that must be taken to avoid the formation of sensitive phases to those agents, during the stainless steel welding procedures, has demanded new stainless steel developments, with higher corrosion resistance, keeping their tensile strength, impact strength and ductility. This is the AISI 904L case, a super austenitic of the latest technology stainless steel for which there is no information about its weldability. Through this research, the weldability and the commercial electrodes most suitable are verified. It was found that when they are applied, the sigma phase is strongly reduced. When this stage appears during the welding procedure, the microstructure formed has a high sensitivity to corrosive agents. It was also found that some electrodes solve this problem and keep the mechanical properties of the analyzed steel.
\end{abstract}

Keywords: Welding, stainless steel, super autenitic, mining.

\section{INTRODUCCIÓN}

Este proyecto tiene su origen en la necesidad de desarrollar tecnología propia para utilizar el acero inoxidable AISI 904L, de muy reciente introducción en los mercados industriales. Estos pertenecen a la familia de los aceros inoxidables austeníticos.

En general, los aceros inoxidables austeníticos contienen Cromo-Níquel en forma importante. Sobre estos se conoce la tecnología para su uso, tanto en los procesos de soldadura, mecanizado y conformado.

Como resultado de estos procesos, quedan "sensibilizados" en el sector adyacente al cordón de soldadura o en el área deformada, manteniendo sus propiedades anticorrosivas en medios no muy agresivos, en cambio, utilizados en medios ácidos, se observa que en la zona "sensibilizada" hay un intenso ataque intergranular que llega a perforar el acero [1].

La razón de esta "sensibilización" esta en el contenido de carbono, el cual al ser calentado, precipita en carburos de cromo, los que se ubican en los bordes de los granos, quedando una estructura cristalina pobre en cromo, pues el precipitado que se forma es del tipo $\mathrm{CCr}$, es decir forma precipitados cuyo contenido en peso en cromo es del 94,3\%.

Dentro de toda la gama de aceros que se ofrecen hoy en día en el mercado, se encuentra el Acero Inoxidable AISI 904L, [2] el cual tiene características mecánicas, físicas y químicas particulares que lo hacen ser único. Este posee una estructura superaustenítica con bajo contenido de carbono y alto porcentaje de Níquel-

\footnotetext{
${ }^{1}$ Universidad Federal del Panamá, Depto. Mecánica, Brasil, ramon @ demec.ufpr.br

${ }^{2}$ Universidad de Tarapacá, Departamento de Mecánica, Facultad de Ingeniería, Arica - Chile, jvillanu@uta.cl

${ }^{3}$ Universidad de Tarapacá, Departamento de Mecánica, Facultad de Ingeniería, Arica - Chile, eponce@uta.cl
} 
Cromo, mejorada la matriz con Molibdeno y Cobre.

El AISI 904L se utiliza principalmente en lugares donde se tiene presencia de soluciones de ácido sulfúrico, fosfórico y/o clorhídrico, en donde otros aceros no han dado resultados satisfactorios [3].

En Chile no se cuenta con la experiencia para soldar el Acero Inoxidable AISI 904L, como tampoco existen publicaciones que indiquen su grado de soldabilidad. Esto es producto de que este acero es también poco utilizado, desconociendo en forma práctica además su grado de conformabilidad. La teoría indica que hay que tomar precauciones para soldar estos aceros, en donde la selección del proceso de soldadura y los consumibles a utilizar formen parte de la realización de una unión soldada libre de defectos [4].

Referente a los consumibles de soldadura, los mercados Nacional e Internacional ofrecen algunas alternativas, de las cuales no se cuenta con experiencia probada de su grado de soldabilidad.

Esta investigación se desarrolla con el objetivo de seleccionar los consumibles con los que se logran las condiciones de anticorrosión, lo más cercanos al material sin soldar.

\section{FUNDAMENTOS TEÓRICOS}

\section{Aceros Inoxidables}

El objetivo de mezclar metales, o metales con no metales es el de obtener aleaciones que puedan ser utilizadas en distintas exigencias.

En el caso del Fe, este material es aleado con otros metales y no metales, con el fin de mejorar sus características mecánicas y/o químicas.

Los elementos de aleación más utilizados son el Silicio, Manganeso, Níquel, Cromo, Molibdeno, Cobre, Vanadio y otros. Algunos de ellos se combinan con el Carbono durante el enfriamiento, formando carburos $\mathrm{u}$ otros compuestos, es decir, son agentes endurecedores del Acero.

Al aumentar a un mínimo de un $12 \%$ de Cromo, se obtienen los aceros inoxidables, los que tienen propiedades mecánicas y químicas especiales. Se acepta que el mecanismo de resistencia a la corrosión en estos aceros, es debido a la formación de una capa superficial de óxido de cromo muy fina, que impide el ataque corrosivo. Para que el acero sea inoxidable, es necesario que concurran las circunstancias de composición, estado del material y medio de ataque convenientes, para que se forme esa capa de óxido protector [5].
En realidad, ninguno de estos aceros es completamente inmune a toda clase de ataques corrosivos. Unos resisten bien a ciertos ácidos y otros a determinadas soluciones, otros resisten el calor, etc. Por eso es conveniente, en cada caso, estudiar cual es el más adecuado.

Además, en el comportamiento de los aceros inoxidables, es muy importante el tratamiento térmico. $\mathrm{Si}$ un acero inoxidable no ha recibido el tratamiento que corresponde, su resistencia a la corrosión puede quedar muy disminuida. En general conviene obtener estructuras ferríticas, martensíticas o austeníticas y evitar la formación de carburos de Cromo, que en estos aceros, elimina la resistencia a la corrosión [6].

\section{Acero Inoxidable Austenítico AISI 904L}

Dentro de la familia de los aceros inoxidables austeníticos se encuentra el AISI904L. Este acero se caracteriza por su alto contenido de Níquel, lo que hace que sea una aleación Níquel - Cromo principalmente, presentando una estructura austenítica a temperatura ambiente.

En el Tabla 1 se presentan las especificaciones de este acero en las normas más comunes y en la Tabla 2 sus componentes [2].

Tabla 1. Comparación de Normas.

\begin{tabular}{|l|l|}
\hline AISI & 904L \\
\hline DIN & X2NiCrMoCu25-20 \\
\hline SIS & 142.562 \\
\hline AFNOR & ZI NCDV 25-20 \\
\hline
\end{tabular}

Tabla 2. Composición Química (\%).

\begin{tabular}{|l|l|}
\hline Carbono (máx.) & 0,02 \\
\hline Cromo & $19-22$ \\
\hline Níquel & $24-27$ \\
\hline Molibdeno & $4-4,8$ \\
\hline Cobre & $1.0-2,0$ \\
\hline Manganeso (máx.) & 2 \\
\hline Silicio (máx.) & 0,5 \\
\hline Fósforo (máx.) & 0,03 \\
\hline Azufre (máx.) & 0,01 \\
\hline
\end{tabular}

Soldabilidad de los Aceros Inoxidable Austeníticos [1], [5], [7], [8], [9].

Estos aceros podrían tener tres tipos de problemas durante la soldadura, estos son: 
- Sensibilización: La precipitación del Cromo en el área adyacente al borde del grano, deja una cantidad insuficiente de Cromo para procurar la mantención de la protección necesaria de la película de óxido.

Existen soluciones para este problema, que es bajar el contenido de Carbono evitando así la precipitación del Cromo. Se considera en la práctica un $0.03 \%$ de Carbono como máximo para prevenir la sensibilización.

Un segundo método de prevención es añadir a la solución Titanio, Niobio o Tantalio. Estos tres elementos tienen alta afinidad con el Carbono y preferentemente forman Carburos, dejando el Cromo en solución.

El último método es raramente usado y consiste en un recocido de la soldadura, una vez finalizada esta. El recocido se hace a una temperatura entre 1038 y $1093^{\circ} \mathrm{C}$, en donde los Carburos que se hubiesen producido, se disolverán. Luego, un enfriamiento rápido, por medio de un templado en agua, los retendrá en solución, previniendo la sensibilización [1], [8] y [9].

- Formación de Fisuras y el Rol de la Ferrita: Bajo condiciones de uniones extremas, a veces se producen fisuras en la soldadura o alrededor de ella, conocidas como fisuras de solidificación y de licuación [10].

Se ha determinado que una pequeña cantidad de Ferrita, alrededor de un 2 a $3 \%$, liberará a la soldadura de las fisuras de solidificación. Principalmente porque la Ferrita aumenta el tamaño límite del grano, aumentando el área disponible de este, lo cual reduce la concentración de segregaciónes de impurezas dañinas, que permanecen dentro de los límites y propicia la nucleación de fisuras.

- Fase Sigma: La fase sigma es un compuesto ínter metálico concentrado de Fierro - Cromo, duro y frágil, que puede ser solamente removido mediante un calentamiento a la temperatura de $1010^{\circ} \mathrm{C}$, en donde este se disolverá (Fig. 1).

La formación de esta fase, se ve favorecida si las partes fabricadas son destinadas para servicios con rangos de $590^{\circ} \mathrm{C}$ a $930^{\circ} \mathrm{C}$, o porque las piezas fabricadas requieren tener un tratamiento térmico de alivio de tensiones sobre estos rangos, reduciendo notablemente su ductilidad. A temperatura de $730^{\circ} \mathrm{C}$ la fase sigma se puede formar rápidamente, pero a bajas temperaturas es necesario un tiempo mayor para su formación. Una variación en la composición, cambiará la velocidad de formación de esta fase y la temperatura a la cual se inicia. El Molibdeno y el Niobio aceleran la reacción sigma, mientras que el Níquel eleva al máximo la temperatura a la cual esta fase aún está presente [1], [5].

En la Fig. 1 se aprecia la fase sigma. Estos son los pequeños trazos rectos y negros que se encuentran en forma desordenada. Además se observa una grieta que cruza la micrografia por el centro.

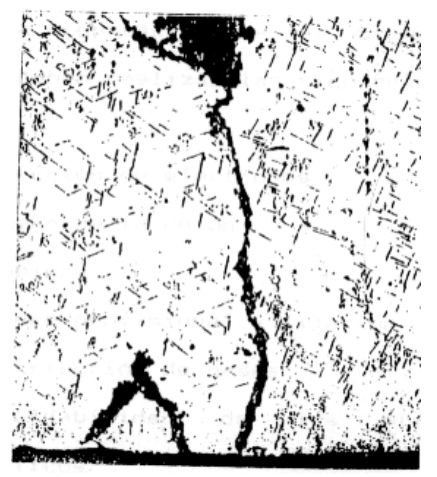

Fig. 1 Fase Sigma (Micrografía 1 (x 400)).

\section{PROCEDIMIENTO TEÓRICO}

\section{Selección de Electrodos}

Para la selección del electrodo se utiliza el diagrama de Schaeffler, (Fig. 2) para aceros inoxidables. Este se utiliza principalmente para predecir la estructura residual del metal obtenido por soldadura en la unión de aceros inoxidables iguales, disímiles o de aceros inoxidables con aceros al carbono con o sin aleación.

Para su empleo se utiliza el Cromo y Níquel equivalente del material base y electrodos. Estos se calculan a partir de las siguientes fórmulas:

$$
\begin{aligned}
& \mathrm{Cr}_{\text {eq }}=\% \mathrm{Cr}+\% \mathrm{Mo}+1,5 * \% \mathrm{Si}+0,5 * \% \mathrm{Nb} \\
& \mathrm{Ni}_{\text {eq }}=\% \mathrm{Ni}+30 * \% \mathrm{C}+0,5 * \% \mathrm{Mn}
\end{aligned}
$$

Cuando se trata de unir materiales de la misma composición química, el punto correspondiente al metal depositado se encontrará entre la recta trazada por los puntos correspondientes al metal base y al electrodo. $\mathrm{Su}$ ubicación específica dependerá del grado de disolución con que se trabaje. En el proceso arco manual el valor típico es un $30 \%$. 
El diagrama de DeLong es una variación del diagrama de Schaeffler, para la determinación de ferrita en la soldadura. DeLong incluyó Nitrógeno en Níquel equivalente. Este diagrama no es adecuado para soldaduras tratadas térmicamente y materiales base afectados por el calor.

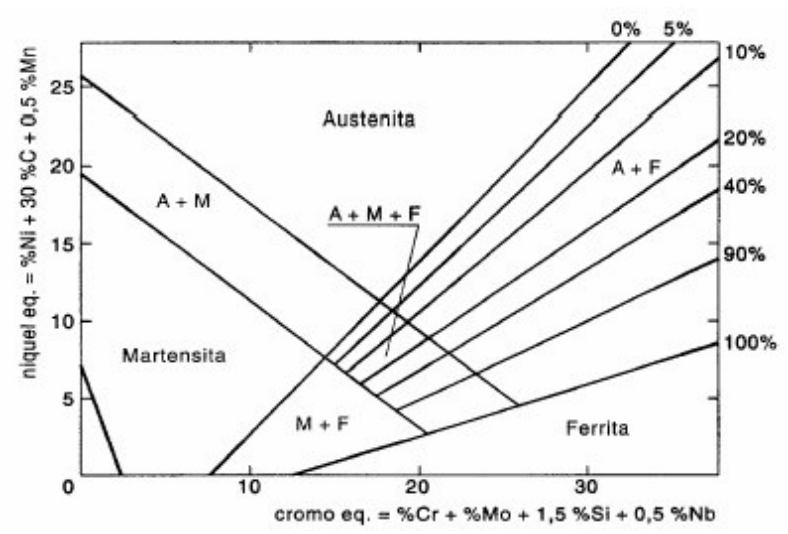

Fig. 2 Diagrama de Schaeffler.

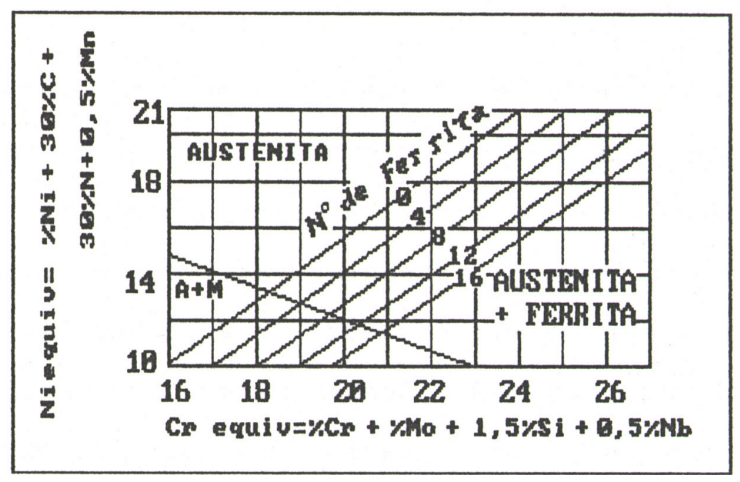

Fig. 3 Diagrama DeLong.

\section{Diseño de la Unión}

El tipo de unión que debe utilizarse, está solamente relacionado con el espesor de las planchas y la disposición de estas. Para ello existen normas para uniones soldadas que definen las dimensiones, para este caso se establecieron las uniones indicadas en la Fig. 4.

\section{Limpieza}

La limpieza que debe hacerse es la usual, para soldaduras de piezas de aceros inoxidables [6]. La Fig. 4 muestra los tipos de unión según el espesor del material.

Unión en forma de I (para espesores hasta $3 \mathrm{~mm}$.). Unión en forma de $\mathrm{V}$ (para espesores hasta $12 \mathrm{~mm}$.). Talón 1 a $2 \mathrm{~mm}$., separación 1 a $2 \mathrm{~mm}$. ángulo de abertura $60^{\circ}$.
Uniones en forma de X. Espesores con más $12 \mathrm{~mm}$.

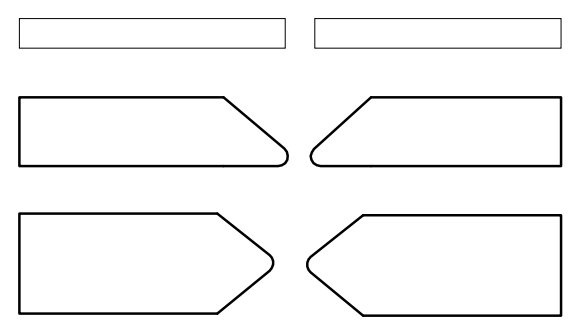

Fig. 4 Tipos de unión según el espesor del material.

\section{Soldadura Provisional y Fijación}

El procedimiento de soldadura en este caso requiere de prensas de fijación, con el fin de mantener rígidas las placas a soldar. Para una mejor comprensión, se presentan en la Tabla 3 las distancias de separación de los puntos de acuerdo a los espesores de plancha y en la Fig. 5 la forma en que estos deben distribuirse.

Tabla 3. Separación de puntos para soldadura.

\begin{tabular}{|l|c|c|c|c|}
\hline Espesor plancha mm. & $1-1,5$ & $2,0-3,0$ & $4,0-6,0$ & sobre 6 \\
\hline Separación ptos. mm. & $30-60$ & $70-100$ & $120-160$ & $150-200$ \\
\hline
\end{tabular}

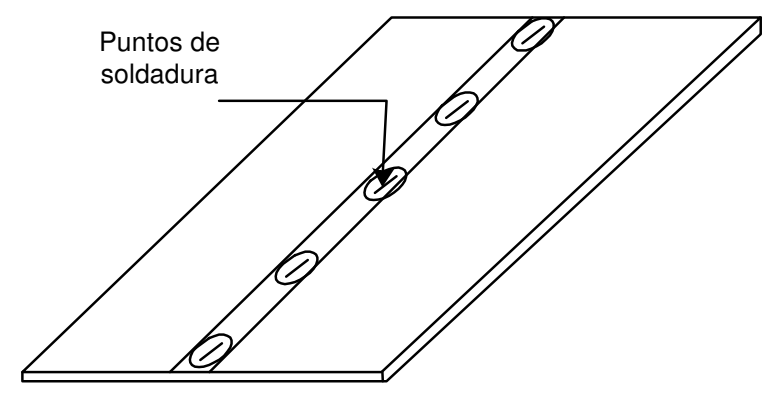

Fig. 5 Representación de puntos para fijación de las planchas.

\section{PROCEDIMIENTO EXPERIMENTAL}

\section{Selección de Consumibles}

La selección de los consumibles se efectúa por medio del diagrama de Schaeffler, que permite determinar los procedimientos de soldadura y los efectos de selección de los parámetros de soldadura en la estructura del cordón de soldadura.

Las composiciones químicas de los electrodos se muestran en la Tabla 4.

Tabla 4. Composición Química de Consumibles. 


\begin{tabular}{|c|c|c|c|c|c|c|c|}
\hline \multirow[b]{2}{*}{ Electrodo } & \multicolumn{7}{|c|}{${ }^{\circ}$ Composición Química \% } \\
\hline & $\mathrm{C}$ & $\mathrm{Cr}$ & $\mathrm{Ni}$ & & Mo & $\mathrm{Cu}$ & $\mathrm{Mn}$ \\
\hline 904L SAM & 0,02 & 20,3 & \multicolumn{2}{|c|}{25,1} & 3,5 & 2 & 2 \\
\hline 904L MIG & 0,013 & 20 & \multicolumn{2}{|c|}{25} & 4,5 & 1,5 & 1,7 \\
\hline 317L MIG & 0,02 & 18 & \multicolumn{2}{|c|}{16} & 4,5 & & 5 \\
\hline $\begin{array}{|ll|}\text { Jungo } & 4500 \\
\text { SAM } & \\
\end{array}$ & 0,025 & 20 & \multicolumn{2}{|c|}{25,5} & 4,8 & 1,5 & 1 \\
\hline $\begin{array}{|ll|}\text { Inconel } & 112 \\
\text { SAM } & \\
\end{array}$ & 0,06 & 21,55 & \multicolumn{2}{|c|}{59,81} & 8,89 & 0,04 & 0,02 \\
\hline \multirow[t]{2}{*}{$\begin{array}{|ll|}\text { UTP } & 1925 \\
\text { MIG } & \\
\end{array}$} & 0,02 & 20 & \multicolumn{2}{|c|}{25} & 4,5 & 1,5 & 1,7 \\
\hline & \multicolumn{7}{|c|}{ Composición Química \% } \\
\hline Electrodo & $\mathrm{Si}$ & $\mathrm{P}$ & $\mathrm{S}$ & $\mathrm{N}$ & $\mathrm{Fe}$ & \begin{tabular}{l|}
$\mathrm{Cb}+$ \\
$\mathrm{Ta}$ \\
\end{tabular} & otros \\
\hline 904L SAM & 0,6 & 0,02 & 0,02 & & & & \\
\hline 904L MIG & 0,4 & & & & & & \\
\hline 317L MIG & 0,4 & & & & & & \\
\hline $\begin{array}{l}\text { Jungo } 4500 \\
\text { SAM }\end{array}$ & 0,9 & & & 0,07 & & & \\
\hline $\begin{array}{l}\text { Inconel } 112 \\
\text { SAM }\end{array}$ & 0,43 & $\begin{array}{l}0,00 \\
6\end{array}$ & $\begin{array}{l}0,00 \\
6\end{array}$ & & 5,83 & 3,36 & 0,5 \\
\hline $\begin{array}{ll}\text { UTP } & 1925 \\
\text { MIG } & \\
\end{array}$ & 0,35 & & & & & & \\
\hline
\end{tabular}

En la Tabla 5, se presentan los porcentajes de Cromo y Níquel equivalentes a los obtenidos de la Tabla 4, utilizando las fórmulas 1 y 2.

Tabla 5. Cu y Ni Equivalentes de Dif. Consumibles.

\begin{tabular}{|l|c|c|}
\cline { 2 - 3 } \multicolumn{1}{c|}{} & $\%$ Cr eq. & \% Ni eq. \\
\hline Metal Base & 25,65 & 27,1 \\
\hline Indura 904L MIG & 25,98 & 25,04 \\
\hline Indura 317L MIG & 24,35 & 14,95 \\
\hline Indura 904L SAM & 25,9 & 27,9 \\
\hline Jungo 4500 SAM & 26,15 & 26,75 \\
\hline Inconel 112 SAM & 32,77 & 61,62 \\
\hline UTP 1925 MIG & 25,03 & 26,45 \\
\hline
\end{tabular}

\section{Montaje de las Planchas}

En la Fig. 6 se observa el montaje de planchas sin respaldo y en la Fig. 7 con respaldo de cobre, con el fin de acelerar el enfriamiento.

\section{Procesos de Soldadura}

Los procesos de soldadura seleccionados son: el Arco Manual y sistema MIG con transferencia Spray, gas de protección de $98 \%$ Ar y $2 \% \mathrm{O}_{2}$.

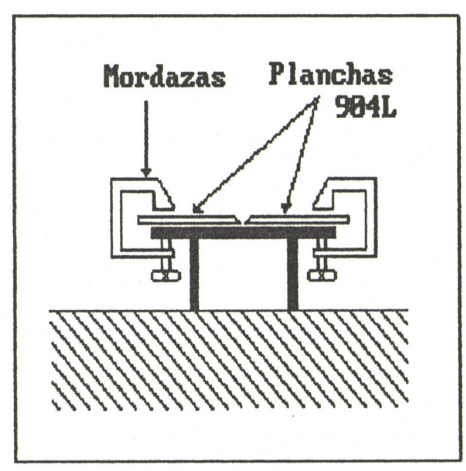

Fig. 6 Montaje sin respaldo de cobre, pero con mordazas para prevenir la deformación.

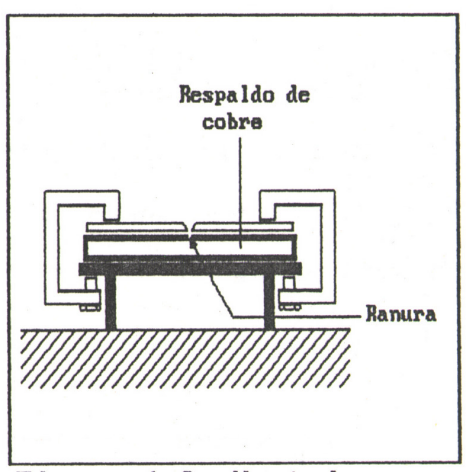

Fig. 7 Montaje con respaldo de cobre para disipar más rápido el calor y guiar al cordón de raíz.

\section{Secuencia de Soldadura}

La secuencia de soldadura utilizada se muestra en la Fig. 8, donde se indican las secuencias de los pases.

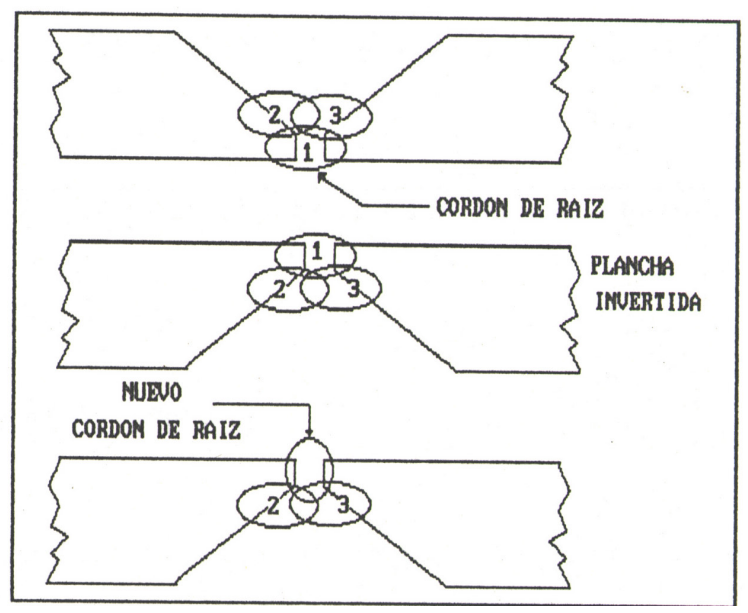

Fig. 8 Secuencia de pases de soldaduras. 


\section{ENSAYOS}

\section{Ensayo de tracción}

Para este ensayo se confeccionan probetas de tracción como se muestran en la Fig. 9 y probetas de Charpy en forma de barra de $7,5 \times 10 \times 55$ con una muesca de $45^{\circ} \times 1$ perpendicular al eje del plano de 7,5.

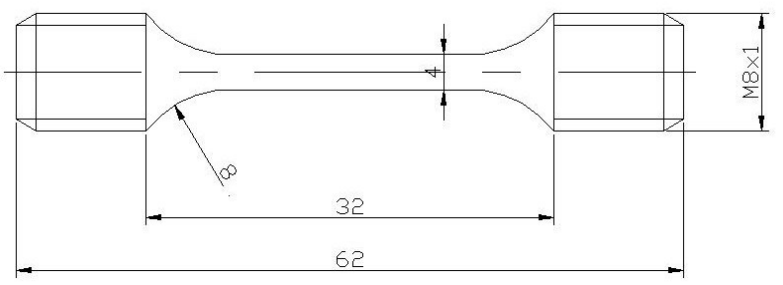

Fig. 9 Probeta de ensayo de tracción.

\section{Ensayo de Doblado}

Se realizaron cuatro probetas de doblado en una prensa cuello de cisne, con ángulos de doblado de $60^{\circ}, 90^{\circ}$, $120^{\circ}$ y $150^{\circ}$.

\section{Ensayo de Corrosión}

Realizados según norma ISO323. Las concentraciones de las soluciones utilizadas en este ensayo, se muestran en el Tabla 6, la solución 0 es el concentrado utilizado en el proceso de lixiviación del cobre, 1, la misma solución duplicada y el 2 , triplicada.

Tabla 6. Solución de lixiviación del Cu.

\begin{tabular}{|l|c|r|c|}
\hline Elemento & 0 & 1 & 2 \\
\hline $\mathrm{Cu}$ & 1.37 & 2.74 & 4.11 \\
\hline $\mathrm{H}_{2} \mathrm{SO}_{4}$ & 17.30 & 34.60 & 51.90 \\
\hline $\mathrm{Fe}^{3+} / \mathrm{Fe}^{2+}$ & 4.0 & 8.00 & 12.00 \\
\hline $\mathrm{Cl}$ & 2.0 & 4.00 & 6.00 \\
\hline $\mathrm{Al}$ & 4.0 & 8.00 & 12.00 \\
\hline $\mathrm{Si}$ & 3.0 & 6.00 & 9.00 \\
\hline $\mathrm{Mn}$ & 2.0 & 4.00 & 6.00 \\
\hline $\mathrm{Mo}$ & 1.5 & 3.00 & 4.50 \\
\hline
\end{tabular}

\section{Limpieza de Probeta}

Antes del ensayo de corrosión, las probetas son decapadas y se eliminan todas las impurezas como fierro embebido, óxidos y grasas. Para esto, se sumergen las probetas en la solución indicada en la Tabla 7 para el fierro embebido y se usa la solución indicada en la Tabla 8 para limpieza y decapado.

\section{Control de Peso}

El control de peso se efectúa en una balanza analógica con una incertidumbre de +/- 0,0005 gr., de acuerdo al cronograma indicado en la Tabla 9.

Tabla 7. Solución Decapante para Fe embebido.

\begin{tabular}{|l|c|}
\hline Agua destilada & $1000 \mathrm{ml}$. \\
\hline Acido nítrico & $30 \mathrm{ml}$. \\
\hline Ferrocianuro de potasio & $30 \mathrm{gr}$. \\
\hline Tiempo de exposición & $10 \mathrm{~min}$. \\
\hline
\end{tabular}

Tabla 8. Solución de limpieza y Decapante.

\begin{tabular}{|l|l|}
\hline Ácido nítrico (76\%) & $25 \%$ \\
\hline Ácido Fluorhídrico & $75 \%$ \\
\hline Tiempo & $45 \mathrm{~min}$. \\
\hline
\end{tabular}

Tabla 9. Peso de Probetas a corroer.

\begin{tabular}{|l|l|}
\hline $1^{\circ}$ Control & Posterior al decapado, inmersión de las probetas \\
\hline $2^{\circ}$ Control & $183 \mathrm{hrs}$. control de peso \\
\hline $3^{\circ}$ Control & $366 \mathrm{hrs}$. control de peso \\
\hline $4^{\circ}$ Control & $597 \mathrm{hrs}$. control de peso \\
\hline $5^{\circ}$ Control & Decapado, control de peso \\
\hline
\end{tabular}

\section{Ensayo de Micrografía}

Para este ensayo se siguen los siguientes pasos:

Pulido Mecánico: Se pule con lijas de 240, 320, 400 y 600 mallas, y finalmente sobre un paño con alúmina, hasta lograr una superficie espejo.

Pulido Electrolítico: Se realiza con la solución indicada en la Tabla 10.

Tabla 10. Solución para pulido electrolítico.

\begin{tabular}{|l|l|l|l|}
\hline $\begin{array}{l}\text { Ácido perclórico } \\
(70 \%)\end{array}$ & $50 \mathrm{ml}$. & $\begin{array}{l}\text { Intensidad } \\
\text { de corriente }\end{array}$ & $6 \mathrm{~A}$ \\
\hline Etanol & $750 \mathrm{ml}$. & Tiempo & $10 \mathrm{seg}$ \\
\hline Agua destilada & $100 \mathrm{ml}$. & \multicolumn{2}{|l}{} \\
\cline { 1 - 2 }
\end{tabular}

\section{Ataque Electrolítico}

Para el ataque electrolítico se utiliza la solución indicada en la Tabla 11. 
Tabla 11. Solución para el ataque electrolítico

\begin{tabular}{|l|l|}
\hline Ácido oxálico & $10 \mathrm{gr}$. \\
\hline Agua destilada & $100 \mathrm{ml}$. \\
\hline Intensidad de corriente & $6 \mathrm{~A}$ \\
\hline Tiempo & $45 \mathrm{seg}$. \\
\hline
\end{tabular}

\section{Nomenclatura de los Ensayos}

Con el fin de identificar claramente las probetas y los ensayos a que estas son sometidas, se crea una nomenclatura de ensayos con dos campos, identificándose en el primer campo el ensayo y en el segundo campo el electrodo utilizado. En la Tabla 12 se muestra la nomenclatura de los ensayos y en la Tabla 13 la identificación de los diferentes electrodos utilizados.

Tabla 12. Identificación de probetas.

\begin{tabular}{|l|l|}
\hline NOMENCLATURA & ENSAYO \\
\hline C & De corrosión (ISO 3231) \\
\hline D & De dureza \\
\hline DB & De doblado \\
\hline T & De tracción \\
\hline CH & De Resiliencia (Charpy) \\
\hline
\end{tabular}

Tabla 13. Identificación de electrodos.

\begin{tabular}{|l|l|}
\hline NOMENCLATURA & ELECTRODO \\
\hline 0 & Metal base 904L \\
\hline 1 & SAM 904L SRC \\
\hline 2 & SAM 904L CRC \\
\hline 3 & MIG 904L SRC \\
\hline 4 & MIG 904L CRC \\
\hline 5 & MIG 317L SRC \\
\hline 6 & MIG 317L CRC \\
\hline 7 & SAM JUNGO 4500 SRC \\
\hline 8 & SAM JUNGO 4500 CRC \\
\hline 9 & SAM INCONEL 112 SRC \\
\hline 10 & SAM INCONEL CRC \\
\hline 11 & MIG UTP 1925 SRC \\
\hline
\end{tabular}

SRC: Soldado s/respaldo de Cu.CRC: Soldado c/ respaldo de Cu.

\section{RESULTADOS DE LOS ENSAYOS}

Las siguientes tablas $15,16,17,18,19,20$ y 21 muestran los resultados de los ensayos.

Tabla 14. Resultados Ensayo de Tracción.

\begin{tabular}{|c|c|c|}
\hline Probetas & $\sigma_{\text {flue }} \mathrm{N} / \mathrm{mm}^{2}{ }^{2}$ & $\sigma_{\max } \mathrm{N} / \mathrm{mm}^{2}$ \\
\hline $\mathrm{T} 0.1$ & 428,8 & 643,2 \\
\hline $\mathrm{T} 0.2$ & 415,9 & 627,4 \\
\hline $\mathrm{T} 1.1$ & 498,2 & 708,7 \\
\hline $\mathrm{T} 1.2$ & 467,3 & 705,4 \\
\hline $\mathrm{T} 3.1$ & 480,5 & 680,1 \\
\hline $\mathrm{T} 3.1$ & 440,7 & 620,2 \\
\hline $\mathrm{T} 5.1$ & 440,7 & 688,7 \\
\hline $\mathrm{T} 5.2$ & 416,2 & 674,1 \\
\hline $\mathrm{T} 7.1$ & 437,7 & 680,4 \\
\hline $\mathrm{T} 7.2$ & 452,0 & 711,5 \\
\hline $\mathrm{T} 9.1$ & 418,6 & 611,4 \\
\hline $\mathrm{T} 9.2$ & 426,9 & 628,0 \\
\hline $\mathrm{T} 11.1$ & 418,6 & 611,1 \\
\hline $\mathrm{T} 11.2$ & 391,7 & 603,9 \\
\hline
\end{tabular}

Tabla 15. Resultados Ensayo Charpy.

\begin{tabular}{|c|c|c|}
\hline Probetas & $\mathrm{E}_{\text {abs }} \mathrm{N}^{*} \mathrm{~m}$ & $\mathrm{R} \mathrm{N} * \mathrm{~m} / \mathrm{cm}^{2}$ \\
\hline CH 0 & 177,2 & 286,9 \\
\hline CH 1 & 89,7 & 146,5 \\
\hline CH 2 & 74,0 & 12,0 \\
\hline CH 3 & 80,4 & 134,9 \\
\hline CH 5 & 133,1 & 212,6 \\
\hline CH 5 & 103,1 & 165,7 \\
\hline CH 6 & 166,6 & 263,8 \\
\hline CH 7 & 58,1 & 103,2 \\
\hline CH 7 & 62,4 & 100,9 \\
\hline CH 8 & 79,2 & 127,2 \\
\hline CH 9 & 46,0 & 77,2 \\
\hline CH 10 & 52,4 & 84,6 \\
\hline CH 11 & 104,9 & 171,2 \\
\hline
\end{tabular}

Tabla 16. Resultados Ensayo de Dureza.

\begin{tabular}{|c|c|c|c|c|c|}
\hline & \multicolumn{5}{|c|}{$\mathrm{N}^{\mathbf{o}}$ de Mediciones (Dureza Hv) } \\
\hline PROB. & 1 & 2 & 3 & 4 & 5 \\
\hline D 0 & 165 & 168 & 174 & 168 & 174 \\
\hline D 1 & 184 & 206 & 218 & 206 & 191 \\
\hline D 2 & 191 & 206 & 202 & 210 & 177 \\
\hline D 3 & 191 & 194 & 181 & 202 & 187 \\
\hline D 4 & 187 & 194 & 187 & 181 & 184 \\
\hline D 5 & 191 & 198 & 214 & 206 & 187 \\
\hline D 6 & 177 & 184 & 214 & 206 & 181 \\
\hline D 7 & 187 & 206 & 232 & 202 & 181 \\
\hline D 8 & 187 & 210 & 218 & 206 & 191 \\
\hline D 9 & 184 & 187 & 247 & 214 & 202 \\
\hline D 10 & 198 & 237 & 237 & 194 & 181 \\
\hline D 11 & 171 & 187 & 198 & 198 & 171 \\
\hline
\end{tabular}


Tabla 17. Resultados Ensayo de Doblado (Dureza Hv).

\begin{tabular}{|c|c|c|c|c|c|}
\hline \multicolumn{6}{|c|}{ Medición Longitudinal } \\
\hline & \multicolumn{5}{|c|}{ PROBETAS } \\
\hline $\mathrm{N}^{\circ}$ MEDIC & DB 1 & \multicolumn{2}{|c|}{ DB 2} & DB 3 & DB 4 \\
\hline 1 & 209,8 & \multicolumn{2}{|c|}{190,8} & 190,8 & 262,9 \\
\hline 2 & 201,9 & \multicolumn{2}{|c|}{183,9} & 190,8 & 235,3 \\
\hline 3 & 201,9 & \multicolumn{2}{|c|}{183,9} & 190,8 & 209,8 \\
\hline 4 & 201,9 & \multicolumn{2}{|c|}{183,9} & 205,7 & 227,1 \\
\hline 5 & 213,88 & \multicolumn{2}{|c|}{201,9} & 241,4 & 227,1 \\
\hline 6 & 227,1 & \multicolumn{2}{|c|}{213,88} & 271,3 & 236,6 \\
\hline 7 & 222,5 & \multicolumn{2}{|c|}{231,7} & 287,3 & 241,4 \\
\hline 8 & 218,2 & \multicolumn{2}{|c|}{236,6} & 323,1 & 241,4 \\
\hline 9 & 213,88 & \multicolumn{2}{|c|}{222,5} & 222,5 & 231,7 \\
\hline 10 & 198 & \multicolumn{2}{|l|}{198} & 209,8 & 222,5 \\
\hline 11 & 205,7 & \multicolumn{2}{|c|}{180,7} & 187,4 & 241,4 \\
\hline 12 & 190,8 & \multicolumn{2}{|c|}{187,4} & 174,3 & 251,1 \\
\hline 13 & 198 & \multicolumn{2}{|c|}{183,9} & 171,2 & 227,1 \\
\hline \multicolumn{6}{|c|}{ Medición Transversal } \\
\hline & \multicolumn{5}{|c|}{$\mathrm{N}^{\mathrm{o}}$ de Mediciones } \\
\hline Probeta & 1 & 2 & 3 & 4 & 5 \\
\hline DB1 & 262,9 & 262,9 & 222,5 & 205,7 & 222,5 \\
\hline DB2 & 323,1 & 257,4 & 231,7 & 227,1 & 241,4 \\
\hline DB3 & 307,8 & 307,8 & 287,3 & 294,1 & 315,3 \\
\hline DB4 & 300,8 & 283,5 & 241,4 & 222,5 & 283,5 \\
\hline
\end{tabular}

DB 1 Doblado a $60^{\circ}$; DB 2 a $90^{\circ}$; DB 3 a $120^{\circ}$ y DB 4 a $150^{\circ}$

Tabla 18. Resultados Ensayo de Corrosión (Pérdida en gramos).

\begin{tabular}{|c|c|c|c|}
\hline Probetas & $0^{*}$ & $1^{*}$ & $2^{*}$ \\
\hline $\mathrm{C} 1$ & 0,001 & 0 & 0,001 \\
\hline $\mathrm{C} 2$ & 0,001 & 0,001 & 0,002 \\
\hline $\mathrm{C} 3$ & 0,003 & 0,002 & 0,001 \\
\hline $\mathrm{C} 4$ & 0,003 & 0,002 & 0,005 \\
\hline $\mathrm{C} 5$ & 0,004 & 0,004 & 0,005 \\
\hline $\mathrm{C} 6$ & 0,004 & 0,003 & 0,003 \\
\hline $\mathrm{C} 7$ & 0,003 & 0,003 & 0,005 \\
\hline $\mathrm{C} 8$ & 0,003 & 0,002 & 0,003 \\
\hline $\mathrm{C} 9$ & 0,003 & 0,003 & 0,002 \\
\hline $\mathrm{C} 10$ & 0,003 & 0,002 & 0,002 \\
\hline $\mathrm{C} 11$ & 0 & 0 & 0 \\
\hline
\end{tabular}

* $\quad 0$ = Solución original; 1 = Solución duplicada,

2 = Solución triplicada
Tabla 19. Parámetros de Soldadura: Velocidad de Avance, Tensión y Corriente.

\begin{tabular}{|c|c|c|c|}
\hline \multirow{2}{*}{ CLASIFICACIÓN } & \multirow{2}{*}{$\begin{array}{c}\text { Velocidad de avance } \\
\mathrm{cm} / \mathrm{seg}\end{array}$} & \multicolumn{2}{|c|}{ CORDON } \\
\hline & & Volts & Amps. \\
\hline $\mathrm{T} 1$ & 0,5 & 15 & 120 \\
\hline $\mathrm{T} 3$ & 0,9 & 24,6 & 168 \\
\hline $\mathrm{T}^{\mathrm{A}}$ & 0,4 & 24 & 165 \\
\hline $\mathrm{T} 4$ & 0,9 & 24,6 & 168 \\
\hline T5 & 1,1 & 24 & 180 \\
\hline $\mathrm{T}^{\mathrm{A}}$ & 0,4 & 24 & 165 \\
\hline $\mathrm{T} 7$ & 0,3 & 14,7 & 110 \\
\hline T9 & 0,4 & 30,6 & 95 \\
\hline $\mathrm{T} 11^{\mathrm{A}}$ & 0,4 & 24 & 165 \\
\hline $\mathrm{C} 1$ & 0,5 & 15 & 120 \\
\hline $\mathrm{C} 2$ & 0,5 & 15 & 120 \\
\hline $\mathrm{C} 3$ & 0,9 & 24,6 & 168 \\
\hline $\mathrm{C} 4$ & 0,7 & 24,6 & 168 \\
\hline $\mathrm{C} 5$ & 0,9 & 24 & 180 \\
\hline C6 & 0,9 & 24 & 180 \\
\hline C7 & 0,5 & 14,7 & 110 \\
\hline $\mathrm{C} 8$ & 0,5 & 14,7 & 110 \\
\hline $\mathrm{C} 9$ & 0,4 & 30,6 & 95 \\
\hline $\mathrm{C} 10$ & 0,3 & 30,6 & 95 \\
\hline $\mathrm{C} 11$ & 0,4 & 24 & 165 \\
\hline $\mathrm{CH} 1$ & 0,5 & 15 & 120 \\
\hline $\mathrm{CH} 2$ & 0,5 & 15 & 120 \\
\hline $\mathrm{CH} 3$ & 0,4 & 24,6 & 168 \\
\hline $\mathrm{CH} 5$ & 1,1 & 24 & 165 \\
\hline $\mathrm{CH} 5$ & 0,4 & 14,7 & 110 \\
\hline CH6 & 0,9 & 24 & 180 \\
\hline $\mathrm{CH} 7$ & 0,5 & 14,7 & 110 \\
\hline $\mathrm{CH} 7$ & 0,3 & 14,7 & 110 \\
\hline CH8 & 0,5 & 14,7 & 110 \\
\hline CH9 & 0,4 & 24 & 165 \\
\hline $\mathrm{CH} 10$ & 0,4 & 30,6 & 95 \\
\hline CH11 & 0,4 & 24 & 165 \\
\hline
\end{tabular}


Tabla 20. Energía Impuesta Promedio.

\begin{tabular}{|c|c|c|c|c|c|}
\hline \multicolumn{6}{|c|}{ ENERGÍA IMPUESTA PROMEDIO KJ / cm } \\
\hline PROBETA & E Prom. & PROBETA & E Prom. & PROBETA & E Prom. \\
\hline T1.1 & 3,8 & CH1 & 3,8 & C1 & 3,8 \\
\hline & & CH2 & 3,8 & C2 & 3,9 \\
\hline T3 & 4,8 & & & C3 & 4,8 \\
\hline T3 & 9,7 & CH3 & 10,1 & C4 & 5,6 \\
\hline & & & & C5 & 5,0 \\
\hline T5 & 11,8 & CH5 & 4,0 & C6 & 5,0 \\
\hline & & CH5 & 6,1 & C7 & 3,5 \\
\hline T7 & 5,6 & CH6 & 4,8 & C8 & 3,7 \\
\hline & & CH7 & 3,8 & C9 & 8,1 \\
\hline T9 & 8,6 & CH7 & 5,6 & C10 & 10,6 \\
\hline & & CH8 & 3,9 & C11 & 12,3 \\
\hline T11 & 12,3 & CH9 & 10,9 & & \\
\hline & & CH10 & 7,9 & & \\
\hline & & CH11 & 12,3 & & \\
\hline
\end{tabular}

Tabla 21. Energía Impuesta Promedio Nº Pases.

\begin{tabular}{|c|c|l|c|c|c|}
\hline \multicolumn{6}{|c|}{ ENERGÍA IMPUESTA PROMEDIO KJ/cm } \\
\hline PROBETA & Pases & PROBETA & Pases & PROBETA & Pases \\
\hline T1.1 & 7,0 & $\mathrm{CH} 1$ & 7,0 & $\mathrm{C} 1$ & 7,0 \\
\hline & & $\mathrm{CH} 2$ & 7,0 & $\mathrm{C} 2$ & 8,0 \\
\hline T3 & 8,0 & & & $\mathrm{C} 3$ & 10,0 \\
\hline $\mathrm{T} 3$ & 4,0 & $\mathrm{CH} 3$ & 4,0 & $\mathrm{C} 4$ & 8,0 \\
\hline & & & & $\mathrm{C} 5$ & 7,0 \\
\hline T5 & 4,0 & $\mathrm{CH} 5$ & 7,0 & $\mathrm{C} 6$ & 7,0 \\
\hline & & $\mathrm{CH} 5$ & 4,0 & $\mathrm{C} 7$ & 9,0 \\
\hline T7 & 5,0 & $\mathrm{CH} 6$ & 8,0 & $\mathrm{C} 8$ & 9,0 \\
\hline & & $\mathrm{CH} 7$ & 9,0 & $\mathrm{C} 9$ & 9,0 \\
\hline T9 & 9,0 & $\mathrm{CH} 7$ & 5,0 & $\mathrm{C} 10$ & 6,0 \\
\hline & & $\mathrm{CH} 8$ & 8,0 & $\mathrm{C} 11$ & 4,0 \\
\hline T11 & 4,0 & $\mathrm{CH} 9$ & 9,0 & & \\
\hline & & $\mathrm{CH} 10$ & 9,0 & & \\
\hline & & $\mathrm{CH} 11^{\mathrm{A}}$ & 4,0 & & \\
\hline
\end{tabular}

\section{ANÁLISIS DE LOS RESULTADOS}

Las probetas que fueron soldadas con respaldo de cobre presentaron falta de fusión en el cordón de soldadura, independiente del proceso de soldadura. Por lo que se analizan sólo las probetas soldadas sin este respaldo.

\section{Ensayo de Tracción}

Soldadura al Arco Manual: Según los resultados de la Tabla 14, la soldadura con el electrodo 904L obtiene valores de esfuerzo de fluencia y esfuerzo máximo mayores que con los demás electrodos, por sobre el metal base, con un módulo de resiliencia alto lo que indica una mayor deformación elástica. Su ductilidad está por debajo del metal base, debido al crecimiento del grano en el área adyacente a la soldadura de la zona afectada por el calor (ZAC).

Los resultados del ensayo de tenacidad muestran que es levemente menor que el material base. La ruptura de la probeta se produce en el metal base y es de tipo semidúctil.

Tanto la ruptura por tracción como la tenacidad confirman el aumento de fragilidad de la ZAC producto del crecimiento del grano, pero zona soldada tiene un comportamiento similar al metal base.

Con el electrodo Jungo 4500, el esfuerzo de fluencia, esfuerzo máximo y resiliencia, son próximos a los valores obtenidos con el electrodo 904L. La tenacidad y la ductilidad presentan valores inferiores al metal base, lo que también fue demostrado en la literatura [11], siendo su ductilidad mayor a los otros electrodos.

Lo único que diferencia a este electrodo del 904L es que contiene $\mathrm{N}$, lo que produce efectos positivos en las propiedades del material, evitando la migración del cromo, traduciéndose esto en la mejora de la ductilidad.

Los resultados obtenidos con el material de aporte Inconel 112 fueron inferiores a los otros electrodos ensayados. Los esfuerzos de fluencia y máximo son menores que los del metal base, módulo de resiliencia igualmente, ductilidad y tenacidad también baja. La fractura se produce en el cordón y es de tipo frágil.

Soldadura Semiautomática MIG: La soldadura con el alambre 904L presenta esfuerzos de fluencia y máximo similares al alambre 317L. Las dos probetas tienen esfuerzos superiores al metal base. Las probetas soldadas con el alambre 317L tienen mayor ductilidad y tenacidad, siendo estas inferiores a las del metal base. En ambos tipos de probetas la ruptura fue justamente en el cordón de soldadura, además fueron soldadas con un alto nivel de energía impuesta.

La ruptura del 904L es del tipo semidúctil, en cambio la del 317L es del tipo frágil. 
La soldadura con alambre UTP 1925 presenta los menores esfuerzos de fluencia y ruptura máxima, por debajo del metal base, teniendo una baja también en la ductilidad y tenacidad. La fractura fue en el cordón de soldadura con característica de fractura frágil.

Ensayo Resiliencia: El material base tiene valores de resiliencia y capacidad para absorber energía superiores a todas las probetas soldadas. Las que fueron soldadas con respaldo, independiente del proceso, tuvieron un valor de resiliencia y capacidad para absorber mayor energía. Sin embargo, es con el proceso MIG que se logran los mayores valores.

La soldadura con el alambre 317L MIG con respaldo de cobre, tiene una resiliencia que es la más próxima al material base, y la resiliencia más baja se obtiene con el electrodo Inconel 112.

\section{Análisis de la Dureza a Través de la Zona Soldada}

Soldadura al Arco Manual: En general en la zona soldada la dureza es superior al material base. En la zona afectada por el calor (ZAC), esta aumenta por los cambios micro - estructurales que producen los ciclos térmicos del proceso.

El electrodo que tiene mayor dureza es el Inconel 112 y el electrodo 904L la más baja. El electrodo Inconel 112 fue soldado con una mayor energía impuesta.

Soldadura Semiautomática MIG: Con este proceso ocurre el mismo fenómeno descrito anteriormente, pero la diferencia de dureza entre las zonas metal - ZAC cordón, son menos pronunciadas. Siendo que no se observa ningún cambio significativo en la dureza al utilizar respaldo de cobre.

\section{Análisis de Probetas de Doblado}

Dureza Longitudinal: Se observa que en estas probetas al acercarse al borde externo, o sea, la zona que sufre una mayor deformación en frío, la dureza tiende a aumentar. También a medida que aumenta el ángulo de doblado la dureza aumenta.

Como se observa en la Tabla 17, la probeta que fue doblada en $120^{\circ}$ presenta una mayor dureza AHe qleresto, sin embargo la probeta de $150^{\circ}$, que es la que tuvo un grado de deformación mayor, muestra una leve disminución de la dqrezzącon respecto a la de $120^{\circ}$.

Dureza Transversal: Todas las probetas siguen una misma tendencia, y es que la dureza en los bordes, es más elevada que la dureza cercana a la línea neutra. En la zona 1 Fig. 17 sometida a tracción los resultados de dureza son parecidos a los obtenidos en la zona de compresión zona 5 Fig. 17. Además se presenta el mismo efecto descrito anteriormente con respecto a la probeta de $120^{\circ}$, la cual dio una dureza mayor que las otras.

Ensayo de Corrosión: De acuerdo a la Tabla 18, no se presenta pérdida de material en las probetas ensayadas. Tanto el material base como el de aporte, se pueden clasificar como materiales sobresalientes para este tipo de soluciones.

\section{Análisis Micrográfico de las Probetas Soldadas}

Las micrografías analizadas son representativas de todos los ensayos realizados y se encuentran caracterizadas y tabuladas con las correspondientes energías impuestas y número de pases, como lo muestran las Tablas 20 y 21 y la Fig. 10 indica las zonas donde se obtuvieron las probetas para obtener las micrografías a analizar.

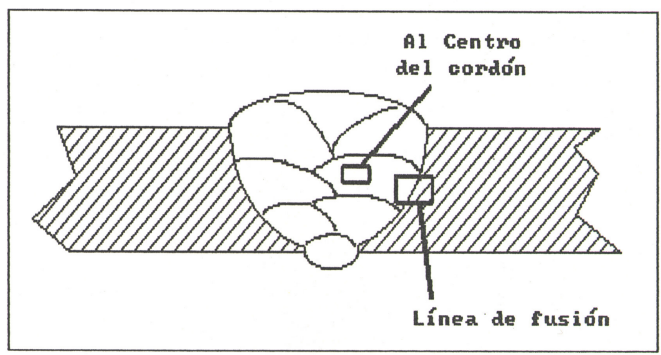

Fig. 10 Zonas de donde fueron retirados las muestras para análisis microscópico.

En la Fig. 11 se observa una matriz completamente austenítica, de grano uniforme con bordes rectos. Se observan líneas de maclas de recocido. La dureza de la matriz es $170 \mathrm{Hv}$ y del grano de austenita es 207,8 Hv. Las franjas horizontales corresponden a bandas de laminación.

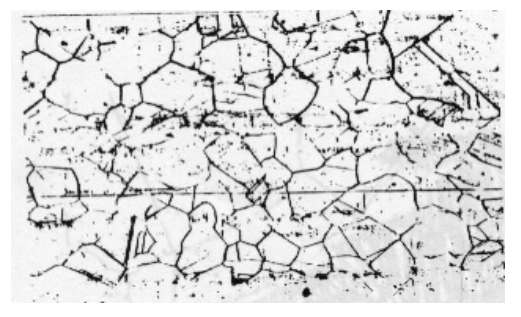

Fig. 11 Micrografía, metal base AISI 904L (x 200). 


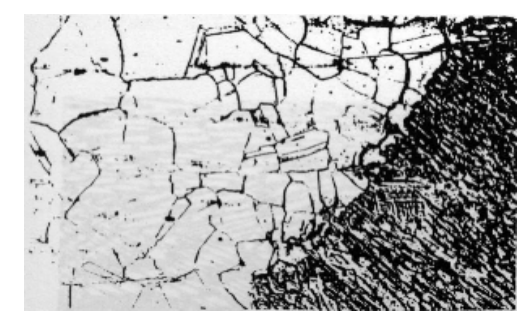

Fig. 12 Micrografía, electrodo Jungo 4500 SAM, línea de fusión (CRC), (x200).

En la Fig. 12 se observa la ZAC con aumento en la dureza $(208 \mathrm{Hv})$ con respecto al metal base $(170 \mathrm{Hv})$.

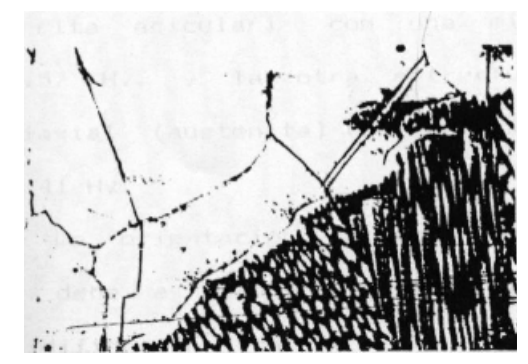

Fig. 13 Micrografía, alambre UTP 1925 MIG. Línea de fusión (SRC), (x400).

En la parte superior de la Fig. 13 se observa el cordón de soldadura, el cual presenta dos distintos tipos de estructura, siguiendo una orientación definida.

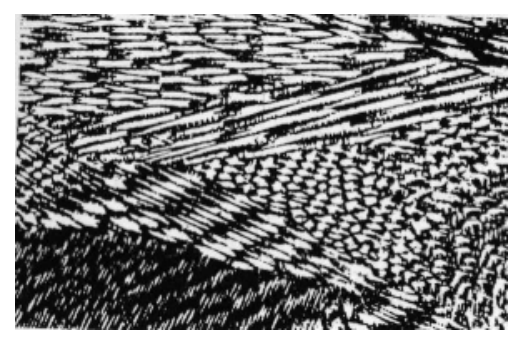

Fig. 14 Micrografía, alambre 904L MIG, cordón de soldadura (CRC), (x100).

La dureza medida en la zona de la Fig. 14 es 187 Hv. Se aprecian dos tipos de formas bien definidas, una de forma laminar (ferrita acicular), con una microdureza de $170 \mathrm{Hv}$, y la otra estructura con forma equiaxial (austenita), con una microdureza de $214 \mathrm{Hv}$.

La orientación y formas de las estructuras se debe a las distintas velocidades de solidificación, en la cual no alcanza a solubilizar en una estructura totalmente austenítica, originándose distintas estructuras intermedias.
En la micrografía de la Fig. 15 se tiene una estructura más grande de forma laminar y una estructura equiaxial mucho más pequeña que en la Fig. 14. Se destacan nítidamente las líneas de estructura laminar y equiaxial. Los conjuntos laminar se diferencian por el tamaño y orientación de las láminas.

Al centro de la micrografía de la Fig. 16 existe una estructura de forma laminar y en los extremos una estructura equiaxial. Los bordes de los granos corresponden a elementos insolubles en la matriz.

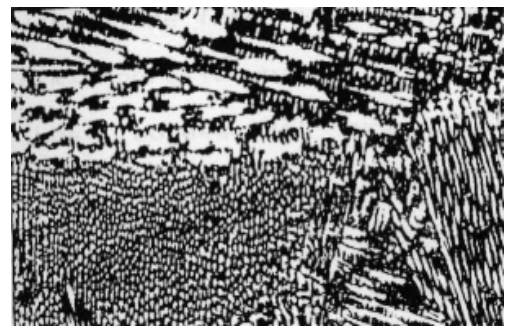

Fig. 15 Micrografía, alambre UTP 1925 MIG, cordón de soldadura (SRC), (x100).

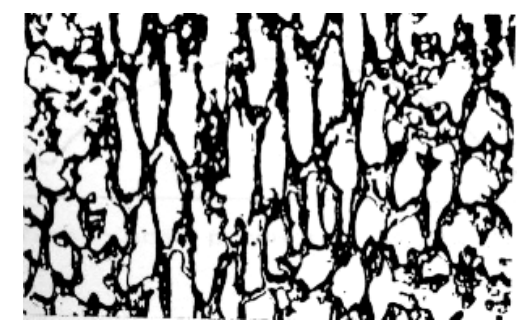

Fig. 16 Micrografía, alambre 904L MIG, cordón de soldadura (CRC), (x1000).

\section{Análisis Micrográfico de las Probetas de Doblado}

En la Fig.17 se presentan las zonas transversales en donde se midieron durezas. Todas las micrografías pertenecen a las zonas 1,3 y 5 . Las bandas negras que aparecen horizontalmente en las micrografías son producto de la laminación del material.

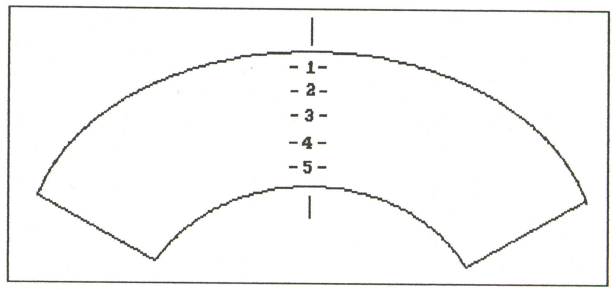

Fig. 17 Zonas transversales de medición de durezas. 
a) doblado a $60^{\circ}$

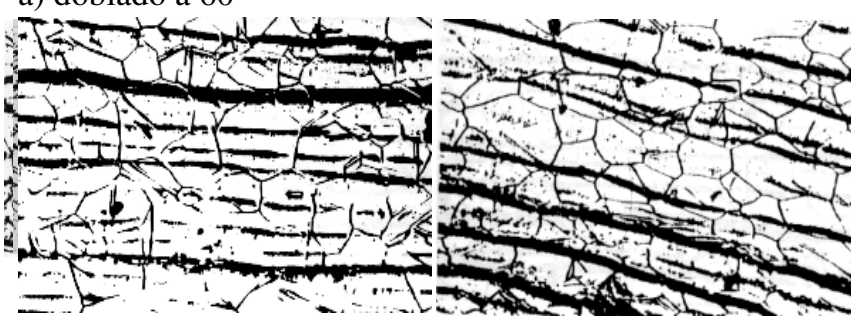

Micrografía 1 (1) x $200 \quad$ Micrografía 2 (3) x 200

Dureza: $263 \mathrm{Hv}$ b) doblado a $90^{\circ}$

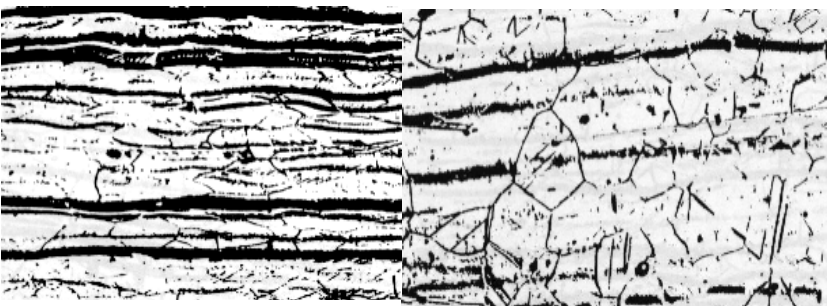

Micrografía 3 (3) x 200

Dureza: $223 \mathrm{Hv}$.
Micrografía 4 (5) x 200

Dureza: $241 \mathrm{Hv}$.

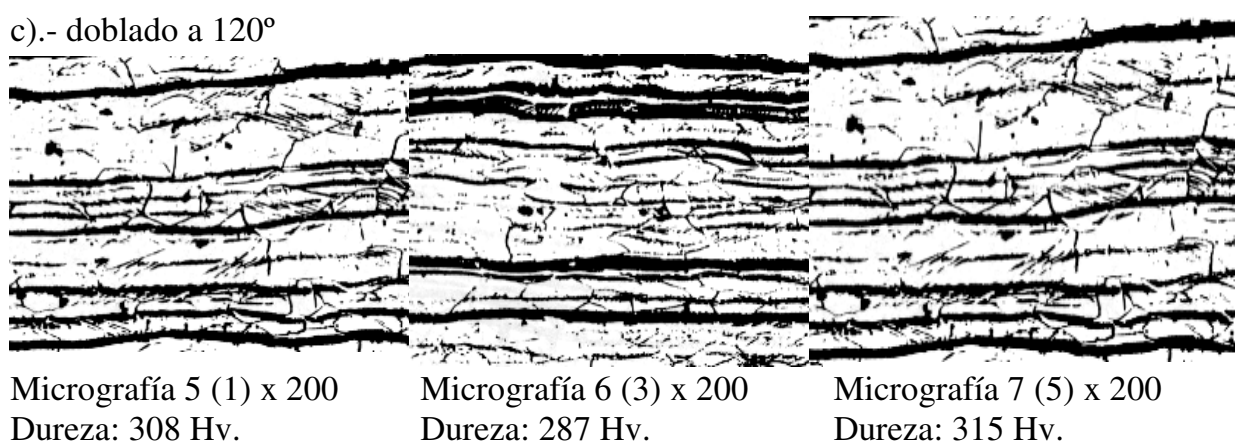

d).- doblado a $150^{\circ}$

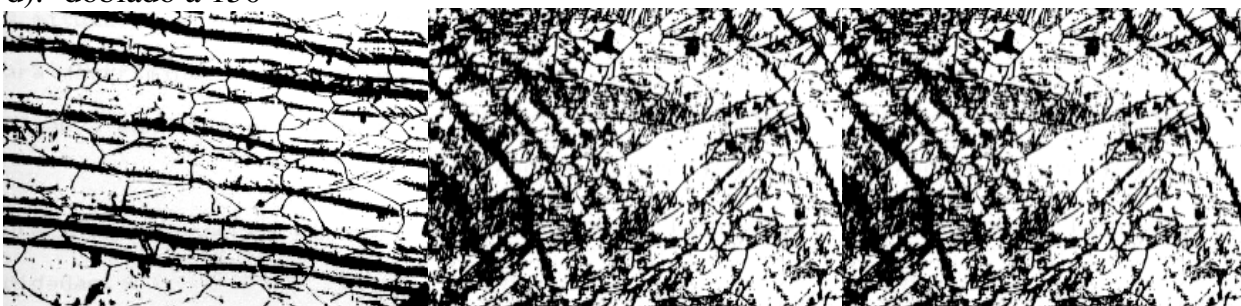

Micrografía 8 (1) x $200 \quad$ Micrografía 9 (3) x 200

Dureza: $301 \mathrm{Hv}$.

Dureza: $241 \mathrm{Hv}$

Micrografía 10 (5) x 200

Dureza: $284 \mathrm{Hv}$.

Fig. 18 Micrografías de doblado: a) doblado a $60^{\circ}$, b) doblado a $90^{\circ}$, c)doblado a $120^{\circ}$ d) doblado a $150^{\circ}$

Zona 1: en esta zona el tamaño de grano se orienta en la dirección de la deformación. Los granos están sometidos a tracción. A mayor ángulo de doblado se aprecia una mayor reducción en el tamaño de grano, como se puede observar en las secuencias de las micrografías, doblada a $60^{\circ}, 5$ doblada a $120^{\circ}$ y 8 doblada a $150^{\circ}$. Esta reducción produce dislocaciones, lo que induce un aumento de la dureza.

Zona 3: en la zona media, el tamaño de grano es más homogéneo que en la zona 1 y 5 , sin ninguna orientación preponderante en las de $60^{\circ}$ y $90^{\circ}$, y leve para $120^{\circ}$ y $150^{\circ}$, como se puede observar en las micrografías 2,3 y 6,9 respectivamente.
Zona 5: en esta zona los granos tienen una forma regular, están sometidas a compresión y se van achicando al aumentar el ángulo de doblado. En la probeta de $150^{\circ}$, micrografía 10 , los granos tienden a deformarse en dirección perpendicular a las líneas de laminación. Es claramente apreciable que al aumentar el ángulo de doblado, micrografía 4 a $90^{\circ}, 7$ a $120^{\circ}$ y 10 a $150^{\circ}$, se obtienen apilamientos y mayor número de líneas de maclas. Dado que las redes cristalinas son comprimidas, estas forman estructuras tetraédricas generándose las mesetas características dentro de los granos. 


\section{CONCLUSIONES}

\section{Ensayos Mecánicos}

El proceso de soldadura afectó negativamente las propiedades de ductilidad y tenacidad, reflejado en la disminución de la resiliencia (Tabla 15) y el aumento de la dureza (Tabla 16) pero mejoró otras como el esfuerzo de fluencia y el esfuerzo máximo, (tabla 14) en el siguiente orden descendente: 904L SAM, JUNGO 4500 SAM, 904L MIG, 317L MIG, Tabla 14.

Se concluye que las soldaduras realizadas con electrodos al arco manual presentan mejores resultados que las soldadas con el proceso semiautomático MIG.

\section{Ensayo de Doblado (Tabla 17)}

El trabajo en frío produce alargamiento de los granos en la parte traccionada. La deformación muy intensa produce una reorientación de los granos hacia una orientación preferente.

La deformación plástica produce un aumento en el número de dislocaciones, lo cual se observa en la dureza en las probetas de doblado, que en virtud de su interacción crean un estado interno de tensiones triaxiales (energía almacenada). Un porcentaje de energía almacenada por la deformación, se aprecia en los defectos de apilamiento y formación de maclas.

A causa de una mayor energía interna del estado de deformación, aumenta la reactividad química, ello conduce a una disminución general de la resistencia a la corrosión y/o posiblemente introduce la posibilidad de formación de grietas por corrosión bajo tensión.

La disminución de dureza que se observa en la probeta de doblado de $150^{\circ}$ (Fig. 18), es debido a que el material llegó a su máxima deformación en frío, en donde su resistencia a la tracción y otros parámetros comienzan a hacerse constantes.

Los procesos de conformado se realizan a velocidades de deformación bajas. A estas velocidades y con los ensayos realizados, no se presenta ninguna transformación de la estructura y no se detectó la formación de collares de precipitado de carburo de Fe en los límites de los granos, como suele ocurrir en los aceros inoxidables austeníticos.

\section{Ensayo de Corrosión}

No se presenta ningún efecto dañino para las probetas. Todas tienen un comportamiento sobresaliente frente al medio corrosivo utilizado, inclusive para ambientes aumentados en concentración, las uniones soldadas y el material base mantienen su comportamiento anticorrosivo como se observa en la Tabla 18.

\section{Micrografías}

La estructura austenítica del material base, no se ven afectadas significativamente por las altas temperaturas que se alcanzan en la ZAC.

Tampoco se observan precipitados de carburos de Cromo en el material base, esto es muy poco probable por su bajo contenido de carbono ( menor que $0,03 \%$ ) y al reducido tiempo en que se mantiene a las temperaturas críticas de sensibilización.

En los cordones de soldadura siempre se presentan dos tipos de estructuras: una acicular y la otra de forma equiaxial.

\section{Calificación de los Electrodos}

En general todos los electrodos presentan un buen comportamiento, sin embargo, aunque todos se comportaron de manera sobresaliente frente a los medios corrosivos, las diferencias se producen frente a la capacidad de absorber energía, esfuerzo de fluencia, esfuerzo máximo o su ductilidad.

\section{Procedimiento de Soldadura}

Los parámetros utilizados en esta experiencia, son los adecuados para la soldadura de este material, sin embargo, la realización de muestras con la variación de estos parámetros permitirá obtener los valores óptimos.

Se presentaron en ciertas probetas una falta de fusión, pero para un soldador con experiencia esto es fácilmente detectable durante el proceso, y con la simple variación de la intensidad de corriente se puede corregir.

La utilización de un respaldo de cobre no presenta ningún beneficio adicional con respecto a las propiedades mecánicas, todo lo contrario, produce defectos en la soldadura que lo hacen innecesario usarlos.

\section{Validez de los Resultados}

Por lo anterior descrito, se verifica que los procesos de soldadura pueden ser utilizados en forma independiente, las diferencias encontradas entre algunos procesos permite seleccionar a priori los parámetros a utilizar para un resultado exitoso. 
Los resultados de dureza son positivos, ya que se obtuvieron durezas superiores a las del metal base Tabla 16. Esta misma situación es válida para la tensión de fluencia, con excepción de MIGM 3 y SAMR 3.

Finalmente, evaluados todos los resultados de Dureza Tracción - Tenacidad, se concluye que los procesos MIGM 3 y SAMR 3 presentan diferencias significativas y menores valores promedios en tracción y tenacidad, por lo cual su utilización es bastante crítica y no se recomienda utilizarlos.

La validez de este estudio está restringida al ámbito de los parámetros analizados, por lo que se recomienda complementarlo considerando otras variables como temperatura, desgaste por roce, etc.

\section{REFERENCIAS}

[1] E. Folkhard; "Welding Metallurgy of Stainless Steels”, Springer Verlar, Wien N.York, 1988.

[2] Loire Creusot; Industrie, División Creusot Marrel.

[3] NACE Standard MR 0175-1990 'Standard Materials Requirements - Sulfide Stress Cracking Resistant Metallic Materials for Oilfield Equipment".

[4] M. Rojas, E. Rojas, R. Cortés; 'Estudio y soldabilidad del acero inoxidable AISI 904L", Tesis de grado, Universidad de Tarapacá, Arica Chile, 1992.
[5] A.F. Padilha, L.C. Guedes; "Aços Inoxidáveis Austeníticos - Microestrutura e Propriedades", Ed. Hemus, Printed in Brazil, 1998.

[6] A. Inchaurza; "Aceros Inoxidables y Aceros Resistentes al Calor", $1^{\text {a }}$ Edición, México, LIMUSA, pp. 25, 1989.

[7] E. Gálvez; "Estudio de Soldabilidad de los Aceros Inoxidables de Uso Industrial", Tesis de grado, Universidad de Tarapacá, Arica - Chile, 1990.

[8] E. Pertenecer et al; "Metalurgia de Soldagem de Aços Inoxidáveis austeníticos com Elevados Teores de Molibdênio", Anais XV Encontro Nacional de Tecnologia de Soldagem, 1999.

[9] P.D. Bilmes; M.J. Solari; 'Metalurgia de la Soldadura y Guías de la Soldabilidad de los Aceros Inoxidables Austeníticos", Anais III Congresso Iberoamericano de Soldagem, 1992.

[10] R. Cortés P., ASCM D' oliveira; 'Trincas de Solidificação e Liquação de Aços Inoxidáveis AISI 904L Soldados por Arco Elétrico", $1^{\circ}$ Congresso Brasileiro de Engenharia de Fabricação. Curitiba PR, 2001.

[11] R. Cortés, P. ASCM D' Oliveira; 'Stainless Steel toughness Super austenitic after Welding", In: International Conference On Advances In Materials And Processing Technologies, Madrid España, 2001. 